\title{
Prevenção de lesões por pressão nos doentes em unidades de cuidados intensivos
}

\author{
Preventing pressure ulcers in patients in intensive care units
}

Prevención de úlceras por presión en pacientes en unidades de cuidados intensivos

\author{
Paulo Alexandre Carvalho Ferreira' ${ }^{\circledR}$; Livia Moreira Delphim ${ }^{\circledR}$; \\ Joana Filipa Cunha Rodrigues' ${ }^{\circledR}$; Maria João da Graça Silva Neves Dias' ${ }^{\circledR}$
}

\begin{abstract}
'Escola Superior de Enfermagem de Coimbra, Portugal
RESUMO

Objetivo: identificar o nível de conhecimento e quais as atitudes dos enfermeiros sobre a prevenção de lesões por pressão nos doentes em unidades de cuidados intensivos. Método: foi realizada pesquisa bibliográfica em abril de 2020, na plataforma EBSCOhost, selecionando as bases de dados Academic Search Complete, CINAHL Complete e MedicLatina, na MEDLINE, através da plataforma Pubmed, na Scopus e na Web of Science. A partir da aplicação dos critérios de inclusão e exclusão, foram analisados oito artigos primários. Resultados: o nível de conhecimento dos enfermeiros demonstrou ser insuficiente e as suas atitudes positivas. Os principais fatores influenciadores foram: idade, anos de experiência profissional, formação/educação e treino na área das lesões por pressão. Conclusão: há necessidade da avaliação do nível de conhecimento dos enfermeiros relativamente à prevenção de lesões por pressão e da criação/implementação de programas educativos. Descritores: Enfermagem; Lesão por Pressão; Cuidados Intensivos; Conhecimento.
\end{abstract}

\section{ABSTRACT}

Objective: to identify nurses' knowledge and attitudes about pressure injury prevention in patients in the intensive care unit. Method: a bibliographic search was carried out in April 2020, in the EBSCOhost platform (selecting the Academic Search Complete, CINAHL Complete and MedicLatina databases), in MEDLINE (through the Pubmed platform), in Scopus and in Web of Science. After application of the inclusion and exclusion criteria, eight primary articles were examined. Results: the nurses' level of knowledge proved to be insufficient and their attitudes, positive. The main influencing factors were age, years of professional experience, education and training in pressure injuries. Conclusion: the nurses' level of knowledge regarding the prevention of pressure injuries needs to be assessed and educational programs, created and implemented.

Descriptors: Nursing; Pressure Ulcer; Intensive Care; Knowledge.

\section{RESUMEN}

Objetivos: identificar el nivel de conocimientos y actitudes de los enfermeros sobre la prevención de úlceras por presión en enfermos en unidades de cuidados intensivos. Método: se realizó una búsqueda bibliográfica en abril de 2020, en la plataforma EBSCOhost, en las bases de datos Academic Search Complete, CINAHL Complete y MedicLatina, en MEDLINE, a través de la plataforma Pubmed, de Scopus y de la Web of Science. A partir de la aplicación de los criterios de inclusión y exclusión, se analizaron 8 artículos primarios. Resultados: el nivel de conocimiento de los enfermeros se mostró insuficiente pero sus actitudes fueron positivas. Los principales factores de influencia fueron la edad, los años de experiencia profesional, la formación/educación y la capacitación en el campo de las úlceras por presión. Conclusión: es necesario evaluar el nivel de conocimiento de los enfermeros en relación con la prevención de úlceras por presión y de la creación/aplicación de programas educativos.

Descriptores: Enfermería; Úlcera por Presión; Enfermería; Cuidados Intensivos; Conocimiento.

\section{INTRODUÇÃO}

As lesões por pressão (LPP) constituem um problema de saúde pública a nível mundial e são um indicador da qualidade dos cuidados de saúde prestados, podendo desenvolver-se em qualquer contexto assistencial. Apesar de $95 \%$ das LPP serem evitáveis através da identificação precoce do risco, este procedimento não é realizado de forma sistemática em todas as unidades de saúde ${ }^{1}$.

Os dados epidemiológicos mais recentes, até à data da pesquisa, relativamente à prevalência de LPP a nível europeu são de 2006, provenientes do estudo realizado pelo Grupo Nacional para o Estudo e Assessoria em Úlceras por Pressão e Feridas Crónicas. Este estudo refere que em ambiente hospitalar a prevalência de LPP varia de acordo com o tipo de unidade de cuidados, sendo que nas $\mathrm{UCl}$ era de $13,2 \%^{2}$.

De acordo com as recentes guidelines do National Pressure Ulcer Advisory Panel (NPUAP), uma LPP é [...] uma lesão localizada da pele e/ou tecido subjacente, normalmente sobre uma proeminência óssea ou relacionada com um dispositivo médico ou outro. (...) A lesão ocorre como resultado da pressão intensa e/ou prolongada ou da pressão combinada com o cisalhamento $[\ldots]^{3: 586}$. 
O enfermeiro tem um papel crucial na prevenção de desenvolvimento de LPP ao colocar em prática as medidas preventivas como a avaliação do risco, posicionamento correto, alternância de decúbito, uso de superfícies de apoio e nutrição adequada. Para que este realize uma correta avaliação do risco, que garanta as intervenções para a prevenção de LPP nos doentes sob os seus cuidados, assim como a seleção das opções terapêuticas adequadas para o tratamento, é fundamental que possua os conhecimentos necessários sobre a etiologia, fisiopatologia das LPP e cuidados preventivos $^{2}$. No entanto, por vezes, parece existir uma lacuna entre o conhecimento científico e a aplicação clínica deste mesmo, tendo em conta as elevadas taxas de incidência e prevalência 4 .

Outro fator que influencia o processo de prevenção de LPP são as atitudes dos enfermeiros. Estas, ao contrário das intervenções, não são observáveis de forma direta, contudo têm um impacto significativo nos comportamentos da pessoa. Assim, as atitudes são variáveis que moldam os comportamentos, sendo importante avaliar as mesmas, permitindo antecipá-los ${ }^{5}$.

De acordo com o European Pressure Ulcer Advisory Panel (EPUAP), o National Pressure Injury Advisory Panel (NPIAP) e a Pan Pacific Pressure Injury Alliance (PPPIA) ${ }^{6}$, as organizações de saúde devem procurar implementar as melhores práticas no contexto clínico. Para tal, o nível de conhecimento dos profissionais de saúde acerca das LPP, assim como as suas atitudes em relação à problemática, devem ser avaliadas, facilitando a implementação de um programa educativo.

A consciência da problemática, uma atitude positiva em relação à prevenção e um adequado nível de conhecimento são os pilares para uma prevenção efetiva, diminuindo o risco de complicações ${ }^{7}$.

A partir do exposto, este estudo possui como questão de revisão: "Qual o nível de conhecimento e quais as atitudes dos enfermeiros sobre a prevenção de LPP nos doentes em UCI?". A partir desta foi elaborado o seguinte objetivo: identificar o nível de conhecimento e quais as atitudes dos enfermeiros sobre a prevenção de LPP nos doentes em UCl.

\section{MÉTOdO}

Este trabalho consiste numa Revisão Integrativa da Literatura (RIL) que reúne a literatura empírica ou teórica existente até ao momento, acerca de um determinado fenómeno ou problema de saúde, de modo a criticar e sintetizar a mesma. Este tipo de literatura apresenta o estado da ciência atual e promove o desenvolvimento teórico ${ }^{8}$.

A elaboração da questão de revisão baseou-se no método PI[C]OD, acrónimo que representa: P - Participantes (Quem foi estudado?); I - Intervenções (O que foi feito?); C - Comparações; O - Outcomes (Resultados, efeitos ou consequências das intervenções) e D - Desenho de estudo (Como a evidência foi recolhida) ${ }^{9}$. Os elementos deste acrónimo são extremamente importantes no processo de construção da questão de revisão, uma vez que permitem maximizar a pesquisa em bases de dados.

O método PI[C]OD inerente à questão de revisão encontra-se descrito na Figura 1.

\begin{tabular}{|c|c|l|}
\hline P & Participantes & Enfermeiros que trabalham nas Unidades de Cuidados Intensivos \\
\hline I & Intervenções & $\begin{array}{l}\text { Avaliação do nível de conhecimento e atitudes sobre a prevenção de } \\
\text { úlceras de pressão }\end{array}$ \\
\hline [C] & Comparações & Não se aplica \\
\hline O & Outcomes & $\begin{array}{l}\text { Nível de conhecimento e atitudes sobre prevenção de úlceras de pressão } \\
\text { em Unidades de Cuidados Intensivos }\end{array}$ \\
\hline D & Desenho do estudo & Estudos primários quantitativos e qualitativos \\
\hline
\end{tabular}

FIGURA 1: método PI[C]OD inerente à questão de investigação. Coimbra, Portugal, 2020.

A RIL foi realizada através da pesquisa bibliográfica no intervalo de tempo de 6 a 10 de abril de 2020, na plataforma EBSCOhost, selecionando as bases de dados Academic Search Complete, CINAHL Complete e MedicLatina, na MEDLINE, através da plataforma Pubmed, na Scopus e na Web of Science, utilizando os Descritores em Ciências da Saúde (DeCS), pressure ulcer, intensive care unit, critical care nursing, prevention, nurse, patients, critical care, knowledge, attitude, perception, e os termos de linguagem natural, bedsore, bed sore, decubitus ulcer, pressure sore, critically ill, wound, nursing care, ICU, intensive care, intensive therapy, intensive therapy unit, critically ill patient, nursing, prevent, preventing, belief, view. Estes foram combinados com os operadores boleanos "AND" e "OR" e utilizadas as truncaturas necessárias para obter uma pesquisa abrangente. 
A expressão de pesquisa utilizada foi a seguinte: (TI/AB) (knowledge* or attitude* or perception* or belief* or view*) $\mathrm{AND}(\mathrm{TI} / \mathrm{AB})$ (nursing or nurse* or "nursing care" or "nursing intervention" or "nursing interventions" or "critical care nursing") AND (TI/AB) (prevention or prevent* or preventing) AND (TI/AB) ("pressure ulcer" or "pressure ulcers" or "pressure sore" or "pressure sores" or bedsore* or "bed sore" or "bed sores" or "decubitus ulcer" or "decubitus ulcers") AND (TI/AB) (ICU or "intensive care unit" or "intensive care units" or "critical care" or "intensive care" or "intensive therapy" or "intensive therapy unit" or "intensive therapy units" or "critically ill" or "critically ill patient" or "critically ill patients").

Com o objetivo de manter a coerência com a questão de revisão foram incluídos nesta investigação: estudos cujos participantes fossem enfermeiros de $\mathrm{UCl}$; cujo fenómeno de interesse fosse o nível de conhecimentos e as atitudes dos enfermeiros sobre a prevenção de lesões por pressão nos doentes em UCl; estudos primários, quantitativos e qualitativos; publicados entre 2015 e 2020; nos idiomas português, inglês, espanhol e francês e disponíveis em texto integral.

Foram excluídos estudos realizados exclusivamente com outros profissionais de saúde ou com enfermeiros em contexto pediátrico.

Com a pesquisa efetuada foi obtida uma amostra inicial de 85 artigos, dos quais foram excluídos 21 artigos duplicados, 49 após aplicação dos critérios de inclusão e exclusão, 3 após leitura do resumo e 4 após leitura integral, obtendo um total de 8 artigos selecionados para a RIL. A análise integral dos artigos foi realizada por dois revisores, de forma independente, de modo a verificar a relevância do estudo. Na Figura 2 encontra-se representado o fluxograma do processo de seleção dos artigos.

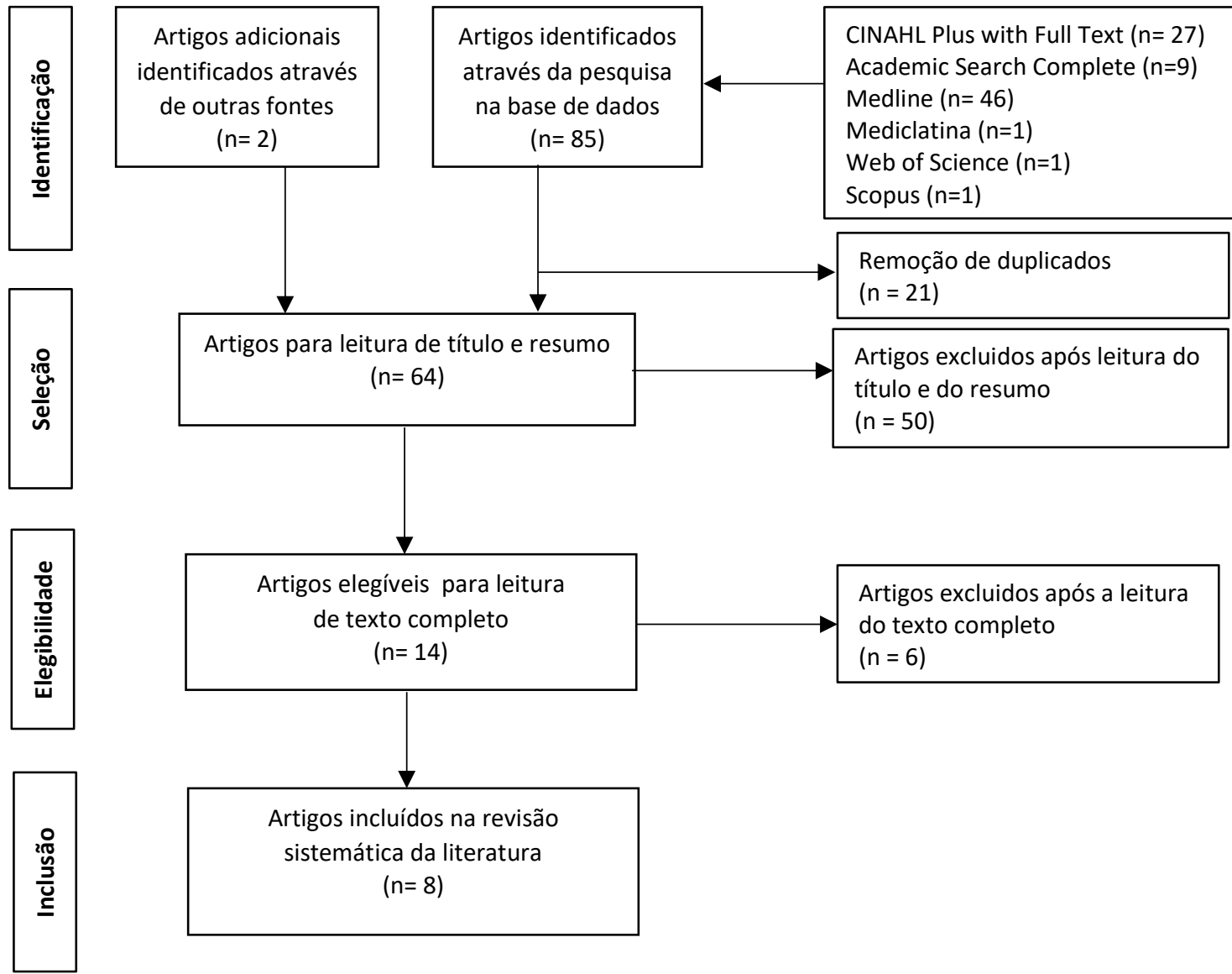

FIGURA 2: Fluxograma do processo de seleção dos estudos. Coimbra, Portugal, 2020.

Investigações referenciadas pelos estudos selecionados, assim como pesquisas não indexadas encontradas pelos investigadores e consideradas pertinentes à temática também foram selecionadas para esta investigação.

Os artigos selecionados encontram-se na Figura 3, estando identificados pelas letras " $\mathrm{C}$ " , " $\mathrm{M}$ ", "S" ou "W", caso tenham sido encontrados na base de dados CINAHL Complete, MEDLINE, Scopus e Web of Science, respetivamente, e numerados por ordem cronológica de publicação. 


\begin{tabular}{|c|c|c|c|c|}
\hline Artigo & Título & Autor & País/ Ano & Periódico \\
\hline $\mathrm{C1}$ & $\begin{array}{l}\text { Knowledge of Nurses Working in Intensive Care } \\
\text { Units in Relation to Preventive Interventions for } \\
\text { Pressure Ulcer }\end{array}$ & $\begin{array}{l}\text { Köse, I, Yeşil, P., Öztunç, } \\
\text { G., \& Eskimez, Z. }\end{array}$ & $\begin{array}{l}\text { Turquia, } \\
2016\end{array}$ & $\begin{array}{l}\text { International Journal of } \\
\text { Caring Sciences }\end{array}$ \\
\hline W2 & $\begin{array}{l}\text { Pressure Injury Prevention in a Saudi Arabian } \\
\text { Intensive Care Unit }\end{array}$ & $\begin{array}{l}\text { Tayyib, N., Coyer, F., \& } \\
\text { Lewis, P. }\end{array}$ & $\begin{array}{l}\text { Arábia } \\
\text { Saudita, } \\
2016\end{array}$ & $\begin{array}{l}\text { Journal of Wound, } \\
\text { Ostomy and Continence } \\
\text { Nursing }\end{array}$ \\
\hline M3 & $\begin{array}{l}\text { Knowledge of the nursing team on pressure ulcer } \\
\text { prevention }\end{array}$ & $\begin{array}{l}\text { Galvão, N. S., Serique, } \\
\text { M. A. B., Santos, V. L. } \\
\text { C. G., \& Nogueira, P. C. }\end{array}$ & Brasil, 2017 & $\begin{array}{l}\text { Revista Brasileira de } \\
\text { Enfermagem }\end{array}$ \\
\hline C4 & $\begin{array}{l}\text { Pressure Injury Prevention: Knowledge and } \\
\text { Attitudes of Iranian Intensive Care Nurses }\end{array}$ & $\begin{array}{l}\text { Tirgari, B., Mirshekari, L., \& } \\
\text { Farouzi, M.A. }\end{array}$ & Irão, 2018 & Wound Care Journal \\
\hline $\mathrm{C5}$ & $\begin{array}{l}\text { Attitudes of intensive care nurses towards } \\
\text { pressure ulcer prevention }\end{array}$ & Habiballah, L. & $\begin{array}{l}\text { Jordânia, } \\
2018\end{array}$ & Clinical Nursing Studies \\
\hline M6 & $\begin{array}{l}\text { Critical Care Nurses' knowledge, attitudes, and } \\
\text { perceived barriers towards pressure injuries } \\
\text { prevention }\end{array}$ & Batiha, A. & $\begin{array}{l}\text { Jordânia, } \\
2018\end{array}$ & $\begin{array}{l}\text { International Journal of } \\
\text { Advanced Nursing Studie }\end{array}$ \\
\hline $\mathrm{C7}$ & $\begin{array}{l}\text { Knowledge and Attitudes Towards Prevention of } \\
\text { Pressure Ulcer: Intensive Care Units Sample in } \\
\text { Turkey }\end{array}$ & $\begin{array}{l}\text { Yilmazer, T., Tüzer, H., \& } \\
\text { Erciyas, A. }\end{array}$ & $\begin{array}{l}\text { Turquia, } \\
2019\end{array}$ & $\begin{array}{l}\text { Turkiye Klinikleri Journal } \\
\text { of Nursing Sciences }\end{array}$ \\
\hline S8 & $\begin{array}{l}\text { Factors related to knowledge, attitude, and } \\
\text { practice of nurses in intensive care unit in the } \\
\text { area of pressure ulcer prevention: A } \\
\text { multicenter study }\end{array}$ & $\begin{array}{l}\text { Khojastehfar, S., Ghezeljeh, } \\
\text { T. N., \& Haghani, S }\end{array}$ & Irão, 2020 & $\begin{array}{l}\text { Journal of Tissue } \\
\text { Viability }\end{array}$ \\
\hline
\end{tabular}

FIGURA 3: Identificação dos artigos selecionados. Coimbra, Portugal, 2020.

\section{RESULTADOS E DISCUSSÃO}

Os oito artigos selecionados consistem em estudos quantitativos descritivos transversais, foram desenvolvidos em contexto de $\mathrm{UCl}$ e os participantes são enfermeiros. Relativamente à metodologia utilizada, todos os estudos aplicaram um questionário composto por vários instrumentos de avaliação, de modo a obter as respostas dos enfermeiros relativamente ao seu nível de conhecimento e atitudes acerca da prevenção de LPP.

Os instrumentos utilizados na maioria dos estudos para avaliar as atitudes e o nível de conhecimento são: o Attitude Towards Pressure Ulcer Prevention (APUP), também referido como Attitude Toward Pressure Ulcer Tool, e o Pressure Ulcer Knowledge Tool (PUKT), também mencionado como Pieper Pressure Ulcer Knowledge Test ou Pressure Ulcer Knowledge Assessment Too/ ${ }^{10-15}$. Todos estes encontram-se validados para os diferentes países nos quais os estudos foram desenvolvidos ${ }^{16}$.

Os artigos M3, C4, M6, C7 e S8 demonstraram que os enfermeiros possuíam um nível de conhecimento insuficiente acerca da prevenção de LPP ${ }^{11-15}$. Estes dados são corroborados em pesquisas prévias realizadas na Bélgica e no Chipre, nas quais os autores concluíram que os enfermeiros possuíam um défice de conhecimento sobre a prevenção de LPP ${ }^{16,17}$. Também outro estudo identificou que os enfermeiros de UCl possuíam um nível de conhecimento insuficiente, sendo a recomendação dos autores a criação de programas educativos para os profissionais acerca da temática ${ }^{18}$. Tal sugestão é reconhecida pelos autores dos artigos $\mathrm{M} 3$ e $\mathrm{M}^{11,13}$.

Esta recomendação está também presente nas guidelines emanadas pelo NPUAP, EPUAP e PPPIA, que referem que as organizações de saúde devem oferecer regularmente formação baseada na evidência científica mais recente sobre LPP, através de programas educativos ${ }^{19}$.

Dos artigos que avaliaram o nível de conhecimento, apenas o artigo C1 revelou que os enfermeiros possuíam um nível de conhecimento suficiente relativamente à prevenção de LPP. Os autores apontam a utilização do instrumento Pressure Ulcer Preventive Interventions Information Form, elaborado pelos mesmos e baseado no Prevention and Treatment of Pressure Ulcers: Quick Reference Guide, desenvolvido pelo EPUAP e o NPUAP, como sendo uma das possíveis causas para os resultados obtidos, uma vez que na maioria da literatura existente o instrumento utilizado é o PUKT, que já se encontra validado em diferentes países, ao contrário do instrumento utilizado no artigo $\mathrm{C}^{20}$.

Foi possível identificar fatores que influenciam o nível de conhecimento, sendo os anos de experiência profissional, a formação/educação e treino na área de LPP transversais aos artigos C1, C4, M6 e S8 ${ }^{12,13,15,20}$. O terceiro fator mais mencionado foi a idade, sendo identificado nos artigos $\mathrm{C} 1$ e C4 como influenciador do nível de conhecimento ${ }^{12,20}$. 
Os níveis de conhecimento mais elevados encontram-se associados a mais anos de experiência profissional, uma vez que proporcionam um maior contacto com a problemática ${ }^{21}$. Também a formação e o treino específico na área de LPP são referidos em outro estudo, no qual é possível verificar que enfermeiros com este tipo de treino possuíam maior nível de conhecimento (51.3\%) comparativamente aos que não tinham realizado o mesmo $(47.7 \%)^{16}$. Relativamente à idade, num estudo realizado no Brasil, os resultados demonstram que o nível de conhecimento sobre prevenção de LPP era superior nos enfermeiros com idade compreendida entre os 30 e 60 anos, comparativamente aos enfermeiros com menos de 30 anos, sendo esta relação evidenciada por um $p$-value $<0.05^{22}$.

Os autores dos artigos W2, C4, C5 e M6 verificaram que os enfermeiros que participaram nos seus estudos possuíam atitudes positivas ${ }^{10,12,13,23}$, contrariamente ao que aconteceu nos artigos $\mathrm{C} 7$ e $\mathrm{S} 8$, onde os profissionais demonstraram ter, na maioria, atitudes negativas ${ }^{14,15}$.

Estudos anteriores, corroboram os resultados obtidos nos artigos W2, C4, C5 e M6, uma vez que as atitudes dos enfermeiros relativamente à prevenção de LPP nestes estudos demonstraram ser positivas ${ }^{5,17}$.

Por outro lado, na literatura, também existem artigos que corroboram os resultados obtidos em C7 e S8, tal como no estudo efetuado na Bélgica, no qual apenas metade dos enfermeiros demonstrou ter atitudes positivas relativamente à temática ${ }^{14-16}$.

Na maioria dos artigos encontrados, os enfermeiros apresentam atitudes positivas, podendo estas diferenças nos resultados derivarem do facto de os instrumentos de avaliação utilizados serem distintos ou serem iguais, mas os seus pontos de corte diferentes ${ }^{14,15}$.

Relativamente aos fatores que influenciam as atitudes dos enfermeiros, os artigos C5 e S8 referem que os participantes do género feminino apresentam melhores atitudes relativamente à prevenção de LPP, assim como aqueles com mais anos de experiência profissional ${ }^{15,23}$. Também os autores do artigo M6 referem que as melhores atitudes são encontradas em enfermeiros que têm mais anos de experiência no geral ou em $\mathrm{UCl}^{13}$. Outro fator apresentado apenas pelos autores do artigo C5 é o facto de os enfermeiros possuírem treino específico recente relativamente à temática ${ }^{23}$.

Os resultados obtidos em estudos anteriores, evidenciam uma correlação positiva entre os anos de experiência profissional e as atitudes em relação à prevenção de LPP ${ }^{24}$, ou seja, será esperado que quanto mais anos de experiência profissional um enfermeiro tiver, mais favoráveis serão as suas atitudes, corroborando os dados obtidos no artigo $\mathrm{M}^{13}$.

Por outro lado, o treino específico recente na área correlaciona-se positivamente com as atitudes dos enfermeiros. No estudo cujos autores chegaram a esta conclusão, os enfermeiros que receberam treino específico na área da prevenção de LPP, no máximo 6 meses antes da aplicação do questionário de avaliação das atitudes, apresentaram maior percentagem no que concerne a atitudes positivas, em comparação com aqueles que o tinham recebido há mais de 2 anos $^{5}$, indo ao encontro do artigo $C 5^{23}$.

Relativamente à relação existente entre o nível de conhecimento e as atitudes, apenas os artigos C4, C7 e S8 analisaram este tipo de relação, ou seja, de que forma o nível de conhecimento pode influenciar as atitudes em relação à prevenção de LPP ${ }^{12,14,15}$. Dos resultados obtidos, foi possível constatar que os artigos C4 e S8 demonstraram existir uma correlação positiva entre as variáveis, apresentando ambos um p-value $<0.001$ (nível de significância p-value $<0,05)^{12,15}$, enquanto o artigo C7 evidenciou o oposto, uma correlação negativa entre as mesmas, ainda que fraca, evidenciada pelo $p$-value $<0.05$ (nível de significância $p$-value $<0,05)^{14}$.

Num estudo sobre o nível de conhecimento e atitudes dos enfermeiros relativamente à prevenção de LPP, os autores constataram a existência de uma correlação positiva entre as variáveis, ou seja, quanto maior for o nível de conhecimento, mais favoráveis serão as atitudes ( $p$-value=0.019). Estes defendem também que aumentando o nível de conhecimento através de programas educativos, existe a possibilidade de melhorar as atitudes dos enfermeiros, o que corrobora a informação anteriormente citada ${ }^{17}$. Os autores do estudo desenvolvido na Bélgica também identificaram uma correlação positiva entre o nível de conhecimento dos enfermeiros e as suas atitudes ( $p-$ value $<0.001$ ), corroborando os dados obtidos nos artigos selecionados ${ }^{16}$.

Por outro lado, o artigo $\mathrm{C} 7$ evidenciou que as atitudes negativas ou inadequadas aumentam com o aumento do nível de conhecimento, sendo este dado justificado pelos autores. Estes afirmam que a correlação identificada é fraca e apontam as caraterísticas individuais de cada um dos enfermeiros como uma possível causa, afirmando que estas são preponderantes para a obtenção de atitudes positivas, em comparação com o nível de conhecimento ${ }^{14}$.

\section{CONCLUSÃO}

É possível concluir que, de modo geral, o nível de conhecimento dos enfermeiros relativamente à prevenção de LPP é insuficiente, sendo os principais fatores que o influenciam a idade, os anos de experiência profissional, a formação/educação e treino na área das LPP. 
A maioria dos enfermeiros demonstrou ter atitudes positivas relativamente à temática em estudo, apresentando como principais fatores influenciadores destas os anos de experiência profissional no geral e em UCl e o treino específico na área da prevenção de LPP.

Tendo em consideração que apenas três dos artigos selecionados avaliaram a existência de correlação entre o nível de conhecimento e as atitudes dos enfermeiros no que diz respeito à prevenção de LPP, em que dois apresentaram uma correlação positiva entre variáveis e o outro uma correlação negativa estatisticamente fraca, não é possível retirar uma conclusão relativamente ao objetivo subjacente. Consideramos, portanto, ser importante o incremento de investigação referente a este ponto específico.

Observa-se a necessidade da avaliação do nível de conhecimento relativamente à prevenção de LPP de forma frequente, de modo a identificar as necessidades dos profissionais, atuando nas mesmas através da elaboração de protocolos onde conste a avaliação da pele, do risco de desenvolver LPP, do risco nutricional, entre outros aspetos, bem como da criação e implementação de programas educativos por parte das organizações de saúde que promovam a prática baseada na evidência científica mais recente. Tendo em conta a especificidade das UCl, será também importante a criação de um grupo de trabalho que apenas desenvolva formações nesta área.

Em suma, a literatura analisada permitiu concluir que os conhecimentos dos enfermeiros, integrados nas categorias sobre a etiologia, classificação e observação, avaliação do risco, nutrição e prevenção de LPP é insuficiente. As atitudes, como a competência pessoal, a responsabilidade e a confiança na efetividade da prevenção, bem como a prioridade dada à prevenção de LPP e a perceção do seu impacto são positivas. Os fatores como: idade, anos de experiência profissional, formação/educação e treino na área de LPP influenciam o nível de conhecimento e as atitudes dos enfermeiros. Por último, não foi possível constatar a existência de uma correlação, positiva ou negativa, entre o nível de conhecimento e as atitudes.

Este trabalho permitiu compreender a importância de manter os conhecimentos dos enfermeiros atualizados, promovendo a consciencialização para a problemática, melhorando, consequentemente, as suas atitudes em relação à mesma.

\section{REFERÊNCIAS}

1. Ministério da Saúde (Pt). Despacho no 1400-A/2015, de 10 de fevereiro. Diário da República, II Série, no 28/2015. Lisboa, 2015 [cited 2020 Aug 11]. Available from: https://dre.pt/pesquisa/-/search/66463212/details/normal?l=1.

2. Afonso $\mathrm{C}$, Afonso $\mathrm{G}$, Azevedo $\mathrm{M}$, Miranda $\mathrm{M}$, Alves $\mathrm{P}$. Prevenção e tratamento de feridas - Da evidência à prática [Internet]. Portugal: Care4wound; 2014 [cited 2020 Aug 15]. Available from: http://www.care4wounds.com/.

3. Edsberg LE, Black JM, Goldberg M, McNichol L, Moore L, Sieggreen M. Revised national pressure ulcer advisory panel pressure injury staging system. Journal of Wound, Ostomy \& Continence Nursing [Internet]. 2016 [cited 2020 Aug 11];43(6):585-97. DOI: https://doi.org/10.1097/WON.0000000000000281.

4. Queiroz Pini LR. Prevalence, risk and prevention of pressure ulcers in units of long-term care [Master's thesis]. Porto, Portugal: Faculdade de Medicina da Universidade do Porto. 2012 [cited 2020 Aug 16]. Available from: https://repositorioaberto.up.pt/bitstream/10216/63858/2/DissertaoLuna\%20PiniUP.pdf.

5. Aslan A, Yavuz van Giersbergen M. Nurses' attitudes towards pressure ulcer prevention in Turkey. Journal of Tissue Viability [Internet]. 2016 [cited 2020 Aug 11]; 25(1):66-73. DOI: https://doi.org/10.1016/j.jtv.2015.10.001.

6. European Pressure Ulcer Advisory Panel, National Pressure Injury Advisory Panel, Pan Pacific. Pressure Injury Alliance. Prevention and treatment of pressure ulcers/injuries: Clinical Practice Guideline EPUAP/NPIAP/PPPIAP; 2019 [cited 2020 Aug 15]. Available from: https://www.cvph.org/data/files/NPIAP\%202019.pdf.

7. Li Z, Zhou X, Cao J, Li Z, Wan X, Li J, et al. Nurses' knowledge and attitudes regarding major immobility complications among bedridden patients: A prospective multicentre study. Journal of Clinical Nursing (John Wiley \& Sons, Inc) [Internet]. 2018 [cited 2020 Aug 11]; 27(9-10):1969-80. DOI: https://doi.org/10.1111/jocn.14339.

8. Carliner S. Workshop in conducting integrative literature reviews. International Professional Communication Conference. 2011 [cited 2020 Aug 11]. Available from: https://ieeexplore.iee.org/document/6087203.

9. Tufanaru C, Munn Z, Aromataris E, Campbell J, Hopp L. Chapter 3: Systematic reviews of effectiveness. In: Aromataris E, Munn Z, editores. JBI Manual for Evidence Synthesis. JBI, 2020. Available from: https://synthesismanual.jbi.global. DOI: https://doi.org/10.46658/JBIMES-20-04.

10. Tayyib N, Coyer F, Lewis P. Pressure injury prevention in a saudi arabian intensive care unit. Journal of Wound, Ostomy \& Continence Nursing [Internet]. 2016 [cited 2020 Aug 11]; 43(4):369-74. DOI: https://doi.org/10.1097/WON.0000000000000245.

11. Galvão NS, Serique MAB, Santos VLCG, Nogueira PC. Knowledge of the nursing team on pressure ulcer prevention. Revista Brasileira de Enfermagem [Internet]. 2017 [cited 2020 Aug 11]; 70(2):294-300. DOI: https://doi.org/10.1590/0034-7167-2016-0063.

12. Tirgari B, Mirshekari L, Forouzi MA. Pressure injury prevention: Knowledge and attitudes of iranian intensive care nurses. Advances in Skin \& Wound Care [Internet]. 2018 [cited 2020 Aug 11]; 31(4):1-8. DOI: https://doi.org/10.1097/01.ASW.0000530848.50085.ef. 
13. Batiha A. Critical care nurses' knowledge, attitudes, and perceived barriers towards pressure injuries prevention. International Journal of Advanced Nursing Prevention [Internet]. 2018 [cited 2020 Aug 11]; 7(2):117-22. Available from: https://www.sciencepubco.com/index.php/IJANS/article/view/22716/11212.

14. Yilmazer $\mathrm{T}$, Tüzer $\mathrm{H}$, Erciyas $\mathrm{A}$. Knowledge and attitudes towards prevention of pressure ulcer: intensive care units sample in Turkey. Turkiye Klinikleri Hemsirelik Bilimleri [Internet]. 2019 [cited 2020 Aug 11]; 11(2):140-7. DOI: https://doi.org/10.5336/nurses.2018-63157.

15. Khojastehfar S, Ghezeljeh TN, Haghani S. Factors related to knowledge, attitude, and practice of nurses in intensive care unit in the area of pressure ulcer prevention: a multicenter study. Journal of Tissue Viability [Internet]. 2020 [cited 2020 Aug 11]; 29(2):76-81. DOI: https://doi.org/10.1016/j.jtv.2020.02.002.

16. Beeckman D, Defloor T, Schoonhoven L, Vanderwee K. Knowledge and attitudes of nurses on pressure ulcer prevention: a crosssectional multicenter study in Belgian hospitals. Worldviews on Evidence-Based Nursing [Internet]. 2011 [cited 2020 Aug 11]; 8(3):166-76. DOI: https://doi.org/10.1111/j.1741-6787.2011.00217.x.

17. Charalambous C, Koulouri A, Roupa Z, Vasilopoulos A, Kyriakou M, Vasiliou M. Knowledge and attitudes of nurses in a major public hospital in Cyprus towards pressure ulcer prevention. Journal of Tissue Viability [Internet]. 2019 [cited 2020 Aug 11]; 28(1):40-5. DOI: https://doi.org/10.1016/j.jtv.2018.10.005.

18. Iranmanesh S, Rafiei H, Foroogh Ameri G. Critical care nurses' knowledge about pressure ulcer in southeast of Iran. International Wound Journal [Internet]. 2011 Oct [cited 2020 Aug 11]; 8(5):459-64. DOI: https://doi.org/10.1111/j.1742481X.2011.00817.x.

19. National Pressure Ulcer Advisory Panel, European Pressure Ulcer Advisory Panel, \& Pan Pacific Pressure Injury Alliance. Prevenção e tratamento de úlceras por pressão: Guia de consulta rápida. NPUAP/EPUAP/ PPPIAP; 2014 [cited 2020 Aug 15]. Available from: https://www.epuap.org/wp-content/uploads/2016/10/portuguese-quick-reference-guide-jan2016.pdf.

20. Köse I, Yeşil P, Öztunç G, Eskimez Z. Knowledge of nurses working in intensive care units in relation to preventive interventions for pressure ulcer. International Journal of Caring Sciences [Internet]. 2016 [cited 2020 Aug 11]; 9(2):677-86. Available from: http://search.ebscohost.com/login.aspx?direct=true \&db=ccm\&AN=117593062\&site=ehost-live.

21. Lawrence $P$, Fulbrook $P$, Miles $S$. A survey of australian nurses' knowledge of pressure injury/pressure ulcer management. Journal of Wound, Ostomy \& Continence Nursing [Internet]. 2015 Sep [cited 2020 Aug 11]; 42(5):450-60. DOI: https://doi.org/10.1097/WON.0000000000000141.

22. Rocha LES, Ruas EFG, Santos JAD, Lima CA, Carneiro JA, Costa FM. Prevenção de úlceras por pressão: Avaliação do conhecimento dos profissionais de enfermagem. Cogitare enferm. [Internet]. 2015 [cited 2020 Aug 11]; 20(3):596-604. Available from: http://search.ebscohost.com/login.aspx?direct=true\&db=ccm\&AN=110567614\&site=ehost-live.

23. Habiballah L. Attitudes of intensive care nurses towards pressure ulcer prevention. Clinical Nursing Studies [Internet]. 2018 [cited 2020 Aug 11]; 6(3):1-7. DOI: https://doi.org/10.5430/cns.v6n3p1.

24. Barakat-Johnson M, Barnett C, Wand T, White K. Knowledge and attitudes of nurses toward pressure injury prevention: A crosssectional multisite study. Journal of Wound, Ostomy \& Continence Nursing [Internet]. 2018 [cited 2020 Aug 11]; 45(3):233-7. DOI: https://doi.org/10.1097/WON.0000000000000430. 